\title{
Cranioplasty with polymethyl methacrylate implant: solutions of pitfalls
}

\author{
Wael M. A. Abd El-Ghani
}

\begin{abstract}
Background: Cranioplasty is important to cover defects following skull surgery and trauma for restoration of function and cosmoses. The objective of the study is to describe the author's technical experience with the use of polymethyl methacrylate (PMMA) implant for cranioplasty to achieve the best results and avoid complications.

Methods: The author describes the indications, locations and operative techniques of cranioplasty using polymethyl methacrylate (PMMA) implant for 75 patients. The implant was used to cover any small- or medium-sized $(<8 \mathrm{~cm})$ cranial defect. Techniques have been done to facilitate reconstruction and to avoid complications to get excellent outcome.
\end{abstract}

Results: This implant has been utilized in cases with excellent cosmetic results and no implant-related complications. Conclusions: Polymethyl methacrylate cranioplasty is a widely performed neurosurgical procedure with good cosmetic outcome and with little related implant complications when done in the proper time and proper techniques.

Keywords: Cranioplasty, Polymethyl methacrylate, Skull defect

\section{Background}

Cranioplasty is the surgical repair of a cranial bone defect, commonly, after a craniectomy or craniotomy for cosmetic and protective effects [1]. This commonly happens when a decompressive craniectomy is needed for brain edema due to traumatic injury, ischemic or haemorrhagic stroke, after the removal of cranio-dural tumors, depressed fractures or even after the correction of skull malformations.

Many materials have been used to repair cranial defects. Ideal material used for cranioplasty would be (1) resistant to infections, (2) not conductive of heat or cold, (3) resistant to biomechanical processes, (4) malleable to fit defects with complete closure and (5) inexpensive [2].

There was an evolution of materials from autologous grafts, allografts and xenografts to a broad spectrum of synthetic materials (metals and acrylics) used for cranioplasty over time [3].

Polymethyl methacrylate (PMMA) is a polymerized ester of acrylic acid discovered in 1939. PMMA is strengthly comparable to the bone. Furthermore, PMMA has better compression and stress resistance than hydroxyapatite; PMMA was shown to adhere to the dura mater without reaction in the underlying tissue $[4,5]$.

Several factors may influence the appearing of complications: materials used, age and general conditions of the patient and the experience of the surgeon on cranial reconstruction. Cranioplasty contraindications include infection, hydrocephalus and brain swelling [6].

This study aimed at achieving the best results and avoiding complications by using polymethyl methacrylate (PMMA) implant for cranioplasty.

\section{Methods}

This study was carried out on 75 patients, 46 males and 29 females, ranging in age from 26 to 60 years (mean $=33$ years); posttraumatic cranioplasty was done at least 1 year after trauma. The site of calvarial defect, indications, possible problems encountered and the surgical technique to solve these problems were studied.

The inclusion criteria are as follows:

Correspondence: waelneuro@yahoo.com

Neurosurgery Department, Assiut University Hospital, Assiut, Egypt 
1. The study included patients with small- and medium-sized bone defects either post-traumatic or as a secondary procedure in some cranial surgeries.

2. After some craniotomies for brain tumors where bone flaps were discarded due to their involvement or due to craniectomy even with the existence of thick protective muscle mass to reduce the incidence of postoperative CSF leak [7].

The exclusion criteria are as follows:

1. Patients with hydrocephalus, infection and brain swelling

2. Children below 4 years old, if there is an intact dura mater, (cranium can achieve self-closure) [3]

All patients were admitted and operated upon in the Neurosurgery Department of Assiut University Hospitals from January 2011 to January 2016.

Patients with post-traumatic bone defects, complicated metal implant and cosmetically disfiguring bone implants underwent 3D-CT scan of skull with measurement of the defect dimensions.

The mean postoperative follow-up period was 13 months with a range of 0 to 45 months. Judgment of the cosmetic results were evaluated by both patient satisfaction and postoperative CT.

\section{Materials and techniques of application}

The Cranioplastic kit used (Teknimed, Biomaterials Innovation, Gentafix $1^{\circ}$, France) is a biocompatible material that is composed of powder and liquid form of polymethyl methacrylate. The powder part consists of polymethyl methacrylate $84.3 \%$, Benzoly peroxide $2.3 \%$, Barium sulphate $9.6 \%$ and Gentamicin sulphate corresponding to $1 \mathrm{~g}$ base with $3.8 \%$ low viscosity. The liquid part consists of PMMA 84.4\%, Butyl methacrylate 13.2\%, $N-N$ dimethyl-p-toluidine $2.4 \%$ and Hydroquinone: $20 \mathrm{ppm}$.

The whole powder and liquid were mixed and prepared to obtain a homogenous mixture, the appropriate quantity of cement was mixed according to the size of the defect, and the final hardening phase takes place in situ.

The mixture was carefully mixed so as to minimize the entrapment of air bubbles and then was left alone. Take the cement in gloved hands and knead it until it no longer adheres to the fingers; insertion in situ can be performed after lying down gel foam over the dura. The impact was inserted and held firmly in place until the cement had been set hard. Any excess cement is removed before hardening. Continuous irrigation with saline is advisable. Hardening time in normal environmental conditions is 10 min. Fixation of the implant was not performed either by titanium screw nor titanium miniplates.

\section{Results}

The indications for cranioplasty in the current study were mainly for reconstruction for bone defects after craniotomies performed for benign brain tumors in 45 cases. As well as, for bone defects after compound depressed skull fractures in 22 cases, five cases were done for replacement for symptomatic or complicated metal implants. Only one case needed cranioplasty to cover defect after cosmetically disfiguring bone implants, another one for replacement of resorbing craniotomy bone flap and one for leptomeningeal cyst (Table 1).

This study described that author has used PMMA to cover suboccipital cranial bone defects in 37 cases, 30 cases were parietal, 3 cases were operated upon for temporal bone defect, two cases were for frontal bone defects and only one case was for occipital bone defect (Table 2).

Table 3 clarifies the size of cranioplasty in relation to location. Suboccipital cranioplasty were operated upon 37 cases (49.3\%) of medium-sized bone defect, parietal cranioplasty was done in 22 cases $(29.3 \%)$ of small size and 8 cases $(10.7 \%)$ were of medium size. In frontal cranioplasty, one case was small size and one was medium. One case was small in temporal cranioplasty and 2 were medium size. The occipital case was medium-sized. Two cases were medium in fronto-tempro-parietal cranioplasty, and no cases were of larger than $8 \mathrm{~cm}$.

Excellent reconstruction was obtained in all cases with no implant-related complications.

\section{Discussion}

Cranioplasty is a commonly performed operation in the field of neurosurgery. The number of craniectomies and the consequent reconstructive procedures has increased during the past decades. Methods of cranioplasty have developed from using autogenous materials to alloplastic materials; the most widely used being is PMMA alone or in combination with titanium or wire mesh [3]. In the current study, the author did not use titanium or wire mesh to reduce infection and cost.

There are many reports about cranioplasty in literatures such as case series, case-control study, cohort

Table 1 Indications for cranioplasty

\begin{tabular}{ll}
\hline Indication & Number \\
\hline Compound depressed skull fractures & 22 \\
Craniotomies performed for benign tumors & 45 \\
Complicated metal implant & 5 \\
Cosmetically disfiguring bone implants & 1 \\
Replacement of resorbing craniotomy bone flap & 1 \\
Leptomeningeal cyst & 1 \\
Total & 75 \\
\hline
\end{tabular}


Table 2 Location of reconstruction cranioplasty

\begin{tabular}{ll}
\hline Location & Number \\
\hline Frontal & 2 \\
Parietal & 30 \\
Temporal & 3 \\
Occipital & 1 \\
Suboccipital & 37 \\
Fronto-tempro-parietal & 2 \\
Total & 75 \\
\hline
\end{tabular}

studies, clinical trials and complications data. Major topics on cranioplasty are such as complications, surgical timing, surgical technique, free flap preservation methods, new materials and cost-effectiveness [8].

Few studies provided surgical basis and techniques that optimize results and reduce complications [3, 9]. In this study, the author describes 75 cases of cranioplasty using PMMA with different indications, sites and sizes as shown in Tables 1, 2 and 3.

The indications for cranioplasty in this study include the following: the size of the defect should be larger than the size of trephine opening (unless in a cosmetically visible location, i.e. forehead), lacks complete covering by thick protective overlying muscle masses, and full thickness scalp covers the area of the cranial defect.

Cranial bone defects that occurred after compound wounds, or where the operative site was infected, are not indicated for cranioplastic repair until at least 1 year has elapsed since compounding or last evidence of wound infection.

In the current study, the policy has been to perform immediate cranioplasty for benign cerebral tumor removals where bone flap is discarded (i.e. meningiomas), or craniectomy is used (i.e. suboccipital craniectomy) without complications.

In the present study, the implant has been used for coverage of small- and medium-sized $(<8 \mathrm{~cm})$ cranial defects in various locations. This experience suggests

Table 3 Size of reconstructive cranioplasty

\begin{tabular}{lll}
\hline Location & Number and size \\
\cline { 2 - 3 } & Small $(<4 \mathrm{~cm})$ & Medium $(4-8 \mathrm{~cm})$ \\
\hline Frontal & 1 & 1 \\
Parietal & 22 & 8 \\
Temporal & 1 & 2 \\
Occipital & 0 & 1 \\
Suboccipital & 0 & 37 \\
Fronto-tempro-parietal & 0 & 2 \\
Total & 75 & \\
\hline
\end{tabular}

that the PMMA implant offers a safe, cosmetically alternative to standard cranioplastic implants.

However, the use of PMMA may be associated with potential complications including an exothermic reaction produced during the curing process which may result in local burn and tissue damage; the author advocates the use of wet gel foam underneath the PMMA together with continuous irrigation with saline to lower the temperature.

The main challenge in the procedure is not in fresh cases such as those after craniotomy for tumors, but it is for cases with trauma or those with thin-scarred scalp. To overcome this problem, the author recommends planning the scalp incision away from the thin-scarred scalp whenever possible. Flap shape needs to be tailored to patient's anatomy considering scalp blood supply, especially in cases where vascularization may be already compromised. In general, it is definitely better to raise a larger flap [10]. Ensure good contouring of the implant. Avoid any prominence whether from the implant or from the surrounding craniotomy. Try to preserve a pericranial flap to form a layer over the implant, this pericranial coverage provides good vasculature for overlying scalp and consequent good wound healing. If good implant contour without prominence is achieved, try not to mobilize it; the best one is the first one. Maintain good homeostasis. Avoid tight head wrapping to maintain good blood flow in the scalp and also avoid very loose wrapping to minimize haematoma collection.

Piitulainen et al. [7] performed cranioplasties with PMMA bone cement, and 5 out of 11 cases had complicated implant; 3 patients presented with surgical site infection, and 2 patients presented with displaced alloplast. "Bone cement reaction" caused by toxicity of methyl methacrylate monomers and exothermic polymerization reaction was one cause for reduced survival of the alloplast.

\section{Conclusions}

Polymethyl methacrylate cranioplasty is a widely performed neurosurgical procedure with good cosmetic outcome and with little related implant complications when done in the proper time and proper techniques.

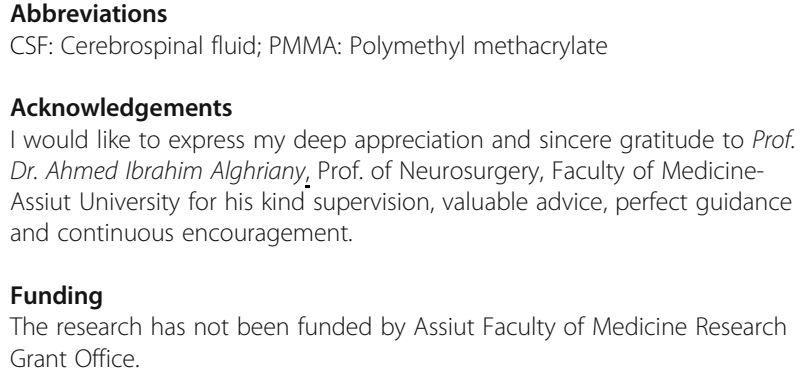

Availability of data and materials

From the corresponding author. 


\section{Authors' contributions}

The author contributed to the protocol development, manuscript writing and editing, data collection and data management. The author read and approved the final manuscript.

\section{Ethics approval and consent to participate}

This work had the approval from Assiut Medical School Ethical Review Board. Informed consent was taken from all individuals who participated in the study.

\section{Competing interests}

The author declares that he has no competing interests.

\section{Publisher's Note}

Springer Nature remains neutral with regard to jurisdictional claims in published maps and institutional affiliations.

Received: 1 October 2017 Accepted: 27 February 2018

Published online: 01 June 2018

\section{References}

1. Sanan A, Haines SJ. Repairing holes in the head: a history of cranioplasty. Neurosurgery. 1997:40(3):588-603.

2. Shah AM, Jung H, Skirboll S. Materials used in cranioplasty: a history and analysis. Neurosurg Focus. 2014;36(4):E19.

3. Aydin S, Kucukyuruk B, Abuzayed B, Aydin S, Sanus GZ. Cranioplasty: review of materials and techniques. J Neurosci Rural Pract. 2011;2(2):162-7.

4. Marchac D, Greensmith A. Long-term experience with methylmethacrylate cranioplasty in craniofacial surgery. J Plast Reconstr Aesthet Surg. 2008;61:744-53.

5. Woodhall B, Spurling RG. Tantalum cranioplasty for war wounds of the skull. Ann Surg. 1945;121:649-68.

6. Grant GA, Jolley M, Ellenbogen RG, Roberts TS, Gruss JR, Loeser JD. Failure of autologous bone-assisted cranioplasty following decompressive craniectomy in children and adolescents. J Neurosurg. 2004;100(2 Suppl Pediatrics):163-8.

7. Piitulainen JM, Kauko T, Aitasalo KM, Vuorinen V, Vallittu PK, Posti JP. Outcomes of cranioplasty with synthetic materials and autologous bone grafts. World Neurosurg. 2015;83(5):708-14.

8. Cho YJ, Kang SH. Review of cranioplasty after decompressive craniectomy. Korean J Neurotrauma. 2017;13(1):9-14.

9. Liu JK, Gottfried ON, Cole CD, Dougherty WR, Couldwell WT. Porous polyethylene implant for cranioplasty and skull base reconstruction. Neurosurg Focus. 2004;16(3):ECP1.

10. Di Rienzo A, Pangrazi PP, Riccio M, Colasanti R, Ghetti I, lacoangeli M. Skin flap complications after decompressive craniectomy and cranioplasty: proposal of classification and treatment options. Surg Neurol Int. 2016; 7(Suppl 28):S737-45.

\section{Submit your manuscript to a SpringerOpen ${ }^{\circ}$ journal and benefit from:}

- Convenient online submission

- Rigorous peer review

- Open access: articles freely available online

- High visibility within the field

- Retaining the copyright to your article 